IRRITABLE BOWEL DISEASE

\title{
Diagnosis of small bowel Crohn's disease: a prospective comparison of capsule endoscopy with magnetic resonance imaging and fluoroscopic enteroclysis
}

\author{
J G Albert, F Martiny, A Krummenerl, K Stock, J Leßke, C M Göbel, E Lotterer, \\ H H Nietsch, C Behrmann, W E Fleig
}

Gut 2005;54:1721-1727. doi: 10.1136/gut.2005.069427

See end of article for authors' affiliations

Correspondence to: Dr W E Fleig, First Department of Medicine, Martin-Luther-University Hospital and Clinics, D-06097 Halle (Saale), Germany; wolfgang.fleig@ medizin.uni-halle.de

Revised version received 24 June 2005 Accepted for publication 27 June 2005 Published online first 14 July 2005

\begin{abstract}
Background and aims: The diagnostic yield of capsule endoscopy (CE) compared with magnetic resonance imaging (MRI) in small bowel Crohn's disease is not well established. We prospectively investigated CE, MRI, and double contrast fluoroscopy in patients with suspected small bowel Crohn's disease.

Methods: Fiffy two consecutive patients (39 females, 13 males) were investigated by MRI, fluoroscopy and-if bowel obstruction could be excluded-by CE. In 25, Crohn's disease was newly suspected while the diagnosis of Crohn's disease (non-small bowel) had been previously established in 27.

Results: Small bowel Crohn's disease was diagnosed in 41 of 52 patients (79\%). CE was not accomplished in 14 patients due to bowel strictures. Of the remaining 27 patients, CE, MRI, and fluoroscopy detected small bowel Crohn's disease in $25(93 \%), 21$ (78\%), and 7 (of $21 ; 33 \%$ ) cases, respectively. CE was the only diagnostic tool in four patients. CE was slightly more sensitive than MRI (12 v 10 of 13 in suspected Crohn's disease and $13 \vee 11$ of 14 in established Crohn's disease). MRI detected inflammatory conglomerates and enteric fistulae in three and two cases, respectively.

Conclusion: CE and MRI are complementary methods for diagnosing small bowel Crohn's disease. CE is capable of detecting limited mucosal lesions that may be missed by MRI, but awareness of bowel obstruction is mandatory. In contrast, MRI is helpful in identifying transmural Crohn's disease and extraluminal lesions, and may exclude strictures.
\end{abstract}

\section{PATIENTS AND METHODS}

\section{Selection of patients}

From 1 May 2002 to 15 December 2003, all patients who were admitted to our hospital for evaluation of suspected or previously diagnosed but worsening Crohn's disease were screened. Crohn's disease was suspected in the presence of suggestive clinical symptoms (diarrhoea, abdominal pain, anorexia, weight loss, rectal bleeding) and biochemical signs of systemic inflammation. Differential diagnoses had been excluded by microbiological stool tests, endoscopy, abdominal ultrasound, and cross sectional imaging.

Eighty one such consecutive patients were identified. They underwent a basic diagnostic workup at our clinic, including abdominal ultrasound, upper endoscopy (optional in established Crohn's disease if performed previously), and ileocolonoscopy. Patients were asked to take part in the study if these tests did not establish a diagnosis other than Crohn's disease or, in patients with previously established Crohn's disease, did not sufficiently explain the clinical situation. Moreover, patients were selected in whom the detection of small bowel involvement was thought to potentially affect treatment strategies.

Exclusion criteria were dysphagia, gastrointestinal obstruction and/or ileus, pregnancy, and the presence of an implanted electromedical device (cardiac pacemaker, defibrillator). Patients under the age of 18 years were also excluded.

The study protocol was evaluated and approved by the ethics committee of the Medical Faculty of the MartinLuther-University. Written informed consent was given by all patients.

Abbreviations: $\mathrm{MRI}$, magnetic resonance imaging; $\mathrm{CE}$, capsule endoscopy; SBFT, barium contrast small bowel follow through; Tlw, $\mathrm{T} 2 \mathrm{w}, \mathrm{T} 1$, T2 weighted; NSAIDs, non-steroidal anti-inflammatory drugs; $\mathrm{CT}$, computed tomography 

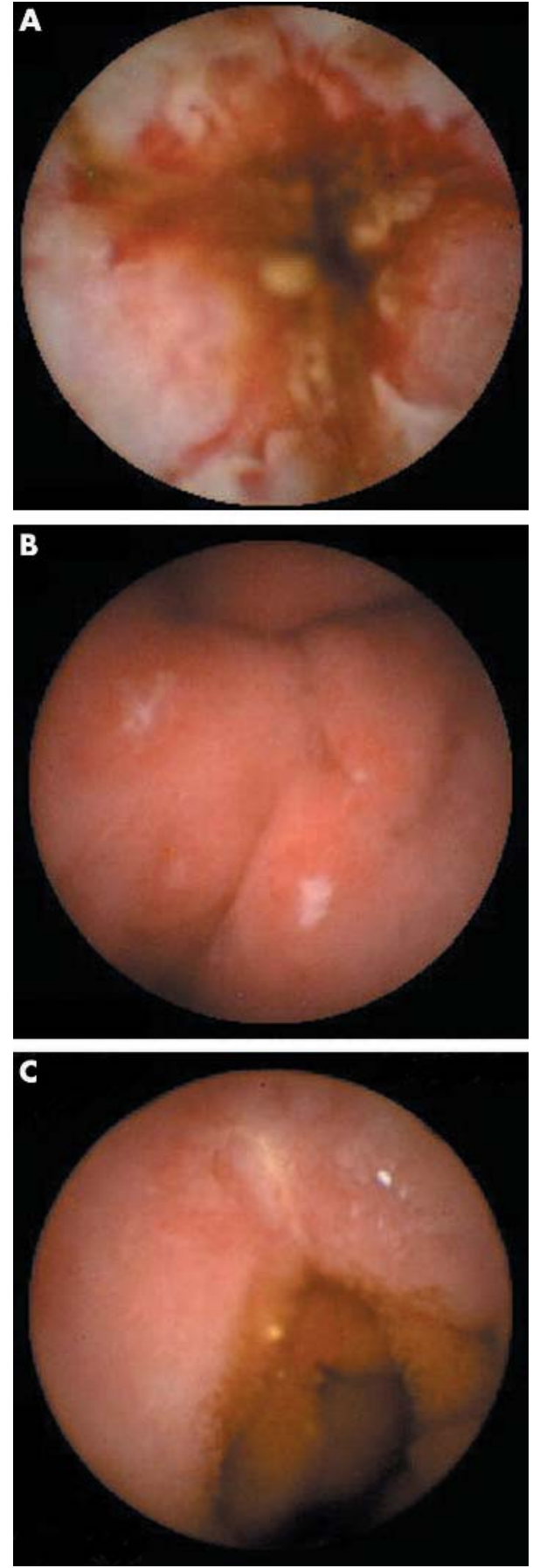

Figure 1 (A-C) Capsule endoscopy findings in small bowel Crohn's disease: ulcerous lesions in the middle small bowel (A), aphthae (B), and fissural lesion (C).

\section{Imaging procedures \\ Capsule endoscopy}

The technique of CE has been described in detail elsewhere. ${ }^{10}$ After an overnight fast, patients ingested $1500-2000 \mathrm{ml}$ of a bowel purgative (Klean-Prep; Norgine, Marburg, Germany). Simethicone (80 mg of Espumisan Emulsion; Berlin-Chemie, Germany) was given with a small amount of tap water $(<20 \mathrm{ml})$ approximately 20 minutes prior to capsule ingestion. ${ }^{11}$ Patients were allowed to drink water after two hours but not to eat until four hours later and were encouraged to walk around if they were able to. Digital video films of the examinations were reviewed using the Rapid Reader 2-software (Rapid Reader; Given Imaging, Yoqneam, Israel). Capsule excretion was reported by the patients themselves within 48 hours. Otherwise, capsule retention was suspected and a plain abdominal radiograph was obtained.

\section{Magnetic resonance imaging}

Patients fasted overnight and drank approximately $1500 \mathrm{ml}$ of a suspension of Klean-Prep one hour before the investigation. Butylscopolaminium bromide (Buscopan $40 \mathrm{mg}$; Boehringer Pharma KG, Ingelheim, Germany) was given intravenously if no contraindications were present. Magnetom Vision and Symphonie scanners (1.5 T; Siemens, Erlangen, Germany) were used to acquire the following sequences: T2 weighted (T2W) coronal and transversal halfFourier turbo spin echo sequence (HASTE; TR $4.4 \mathrm{~ms}$; TE $64.0 \mathrm{~ms}$; slice $9 / 8 \mathrm{~mm}$ ), T2 $\mathrm{w}$ transversal IRM (TR $1000 \mathrm{ms,}$ TE $62 \mathrm{~ms}$ ), abdominal and pelvic projections, "thin slice" RARE (TR 11.9 ms; TE 95 ms; TD 0; slice 64 mm; ETL 240), and Tlw flash two dimensional coronal and transversal sequences (abdomen: TR 147.2/115 ms; TE $2.3 \mathrm{~ms}$; flip 70 8 / $5 \mathrm{~mm}$; pelvis: TR $191 \mathrm{~ms}$; TE $2.5 \mathrm{~ms} ; 7 \mathrm{~mm}$ ) with fat saturation, in abdominal and pelvic projections. Tlw images were obtained before and after intravenous administration of $0.1 \mathrm{mmol} / \mathrm{kg}$ body weight gadolinium chelate (Magnevist; Schering, Berlin, Germany).

\section{Enteroclysis}

Patients fasted overnight. They were intubated nasally with a duodenography catheter, the tip of which was advanced to the proximal jejunum distal to the ligament of Treitz. Approximately 140-200 ml of barium sulphate suspension was injected into the small bowel under fluoroscopy. Thereafter, methyl-cellulose solution was continuously injected to obtain double contrast images. Radiographs in the prone, supine, and oblique positions were obtained. ${ }^{12}$

\section{Sequence of investigations}

To exclude bowel strictures, enteroclysis and/or MRI were performed prior to CE. Significant bowel stricture was defined as luminal narrowing to less than $12 \mathrm{~mm}$. CE was not performed in these cases. The examiners of CE, MRI, and enteroclysis were blinded to each others findings but were aware of patient history and laboratory data.

\section{Outcomes}

The primary event of interest was detection of an inflammatory lesion of the small bowel. In addition, complications, patients' acceptance of the diagnostic methods, and final diagnosis after 12 months of follow up were investigated.

In CE, detection of aphthous mucosal lesions, irregularly shaped or fissural ulcers (occasionally associated with bleeding), cobblestone appearance, luminal narrowing due to oedema and/or fibrous scarring, and granularity with attenuated or lost vascular pattern resulted in the diagnosis of Crohn's disease (fig 1). On the other hand, findings such as patchy mucosal erythema, oedema, or a single regular ulceration were considered inconclusive.

MRI features indicative of active small bowel Crohn's disease included thickening of the bowel wall $(\geqslant 4 \mathrm{~mm})$ and enhancement of the bowel wall after application of intravenous contrast medium (fig 2). In contrast, weak enhancement of bowel loops without bowel wall thickening was interpreted as an non-specific finding. All investigations were accomplished within 10 days, with the exception of one patient (six weeks).

Patient comfort during the imaging procedures was determined by use of a questionnaire which was to be completed within five days of the procedure. Patients were asked to grade "stress" on a scale of 0 (no stress at all) to 10 

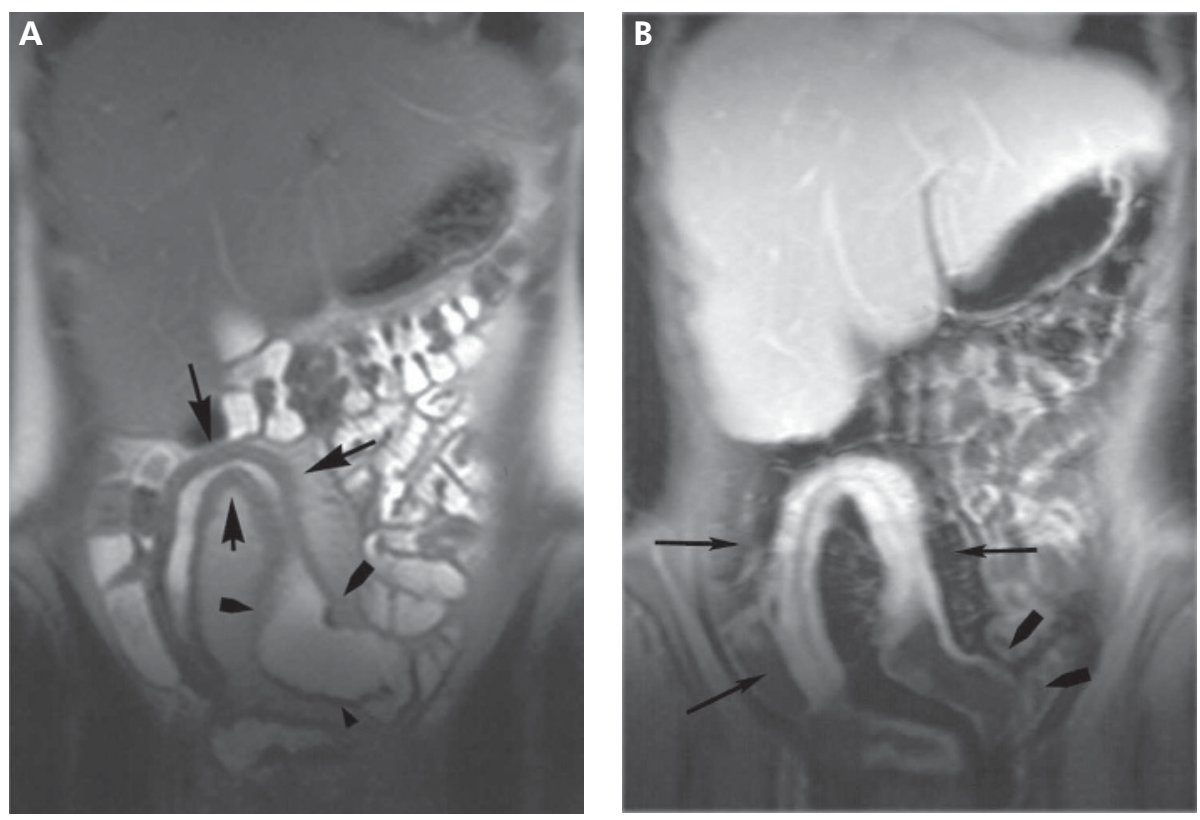

Figure 2 (A, B) Magnetic resonance imaging findings. Inflammation of a distal small bowel segment (A, B) and enhancement after application of contrast medium (B). Subcritical stricture with slight dilation of proximal bowel segment (arrowheads).

(unbearable stress) for each of the imaging procedures (MRI, $\mathrm{CE}$, and enteroclysis).

None of the patients was receiving regular non-steroidal anti-inflammatory drugs (NSAIDs). In addition, all patients denied the occasional intake of NSAIDs during the study.

\section{Statistical analysis}

Continuous variables are expressed as means (SD) and categorical variables as percentages. Categorical variables were compared using Fisher's exact test. A p value of less than 0.05 was considered statistically significant. To account for multiple testing of outcome data arising from individual patients, Bonferroni's correction was used where appropriate, and significance was only asserted with $\mathrm{p}<0.01$ for an individual test. The Wilcoxon test was used for comparing patients' acceptance of the investigations. The McNemar's test was used to quantify interobserver agreement, and chance corrected index ("kappa statistics") was given in this case. WinStat 3.1 for MS Excel, version 2003.1 (Robert K Fitch Software, Germany) software was applied.

\section{RESULTS}

\section{Patients}

Eighty one consecutive patients were screened for eligibility. In 28 of these patients, either a definitive diagnosis was made by basic procedures or it was determined that clinical management would not be affected by potential small bowel involvement. In one case, urgent surgical intervention was necessary. Thus 52 patients were enrolled ( 39 females, 13 males) aged 18-72 years (mean 36.6 (SD 12.41) years for males and 39.7 (16.0) years for females) (fig 3).

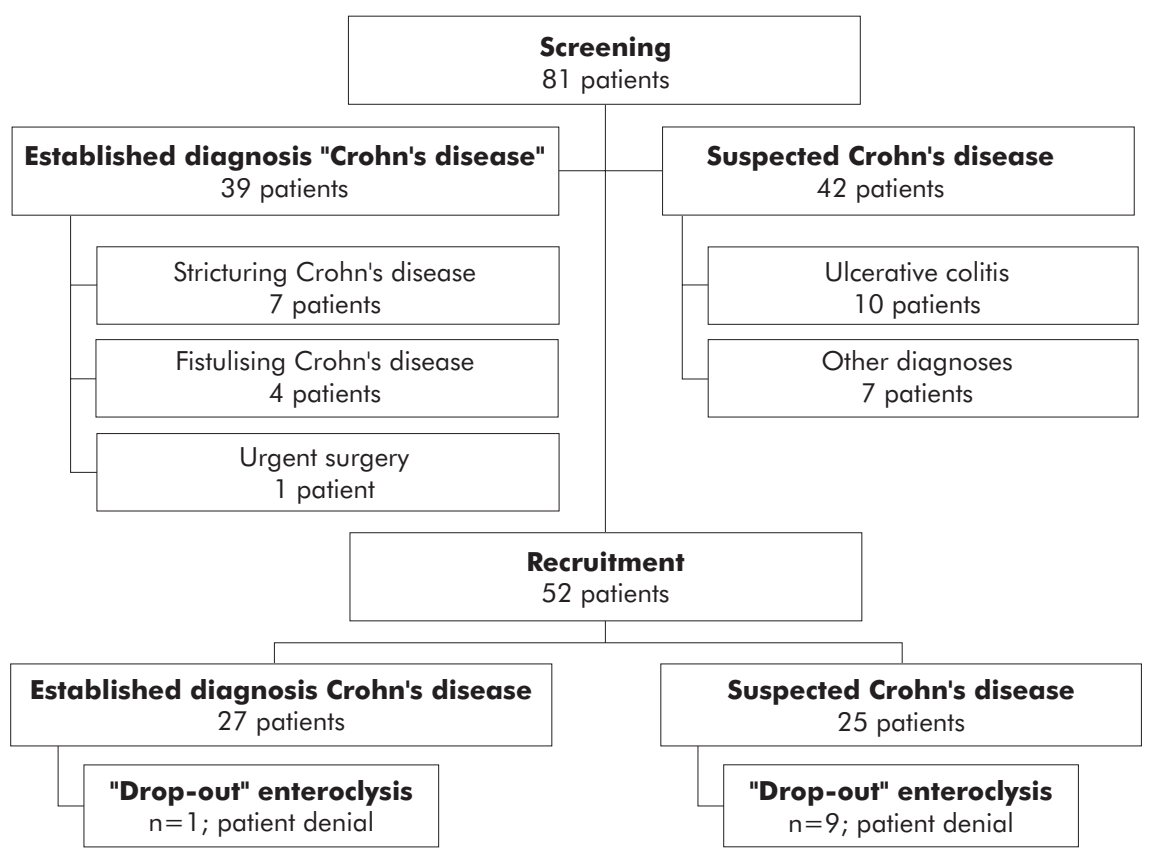

Figure 3 Flow chart of the study group. 
Twenty five patients were newly suspected to have Crohn's disease while a diagnosis of Crohn's disease had been previously established in 27 . The leading symptom at hospital admission was abdominal pain in $27 / 52$ patients $(51.9 \%)$, diarrhoea in $19(36.5 \%)$, and weight loss or perineal fistula with suspected Crohn's disease in three patients each (5.8\%). In patients with established Crohn's disease the diagnosis had been made an average of 8 years (range 6 months to 23 years) previously. Sixteen of these patients had undergone bowel surgery 7-172 months before inclusion in the study (mean 66.5 (48.34) months). Surgical interventions comprised ileocaecal resection $(n=7)$, resection for stricture or strictureplasty (5), segmental resection for fistula (2), and partial colectomy (2).

Findings in patients with established Crohn's disease In patients with an acute flare of established Crohn's disease, enteroclysis detected small bowel lesions in 16 of 27 cases $(59.2 \%)$. No lesion was found in three cases. High grade bowel stricture was proven by enteroclysis in 12 cases.

MRI detected inflammatory small bowel lesions in 22 of 27 patients $(81.5 \%)$. One patient did not tolerate the procedure of enteroclysis but MRI detected a high grade bowel stricture.

Hence CE was not performed in 13 patients due to bowel stricture. In the remaining 14, typical features of small bowel Crohn's disease were detected in all but one patient (92.9\%). In this patient only non-active supposedly post-inflammatory "breaking" of the mucosal folds was reported. In direct comparison, CE found slightly more inflammatory lesions than MRI; the difference was not statistically significant $(\mathrm{p}>0.05)$ (table 1).

CE was the exclusive diagnostic tool in two patients. In a 23 year old female, ileocolonoscopy showed normal colonic and ileal mucosa, and MRI detected some non-specific contrast enhancement in the pelvic small bowel without thickening of the bowel wall. CE demonstrated fissural and irregular ulcerous lesions, granularity, and loss of vascularity of the middle small bowel, and the capsule was retained in a functionally stenotic area for approximately 30 minutes. Enteroclysis had been previously refused by the patient because of radiation exposure. The second patient was a 32 year old male with a weight loss of $10 \mathrm{~kg}$. Both enteroclysis and MRI found a non-significant narrowing of the anastomosis that was present after segmental colectomy four years earlier. MRI suggested the luminal narrowing to be non-inflammatory in nature and showed minor contrast enhancement of the proximal small bowel without wall thickening. CE disclosed discontinuous aphthous disease of the entire small bowel. Anti-inflammatory therapy was initiated.

Findings in patients with suspected Crohn's disease Of 25 patients with suspected Crohn's disease, the diagnosis was confirmed in 14 (56\%) and rejected in 11 (44\%) (table 2).

Small bowel lesions were detected by enteroclysis in four of 14 patients with a final diagnosis of Crohn's disease (28.6\%).

Table 1 Capsule endoscopy (CE) compared with magnetic resonance imaging (MRI) in patients with a previously established diagnosis "Crohn's disease"

\begin{tabular}{lll}
\hline \multicolumn{3}{c}{ Small bowel lesions detected } \\
\hline$C E$ & $13 / 14$ & $92.9 \%$ \\
MRI & $11 / 14$ & $78.6 \%$ \\
\hline$p>0.05$, kappa $=0.44$ \\
\hline
\end{tabular}

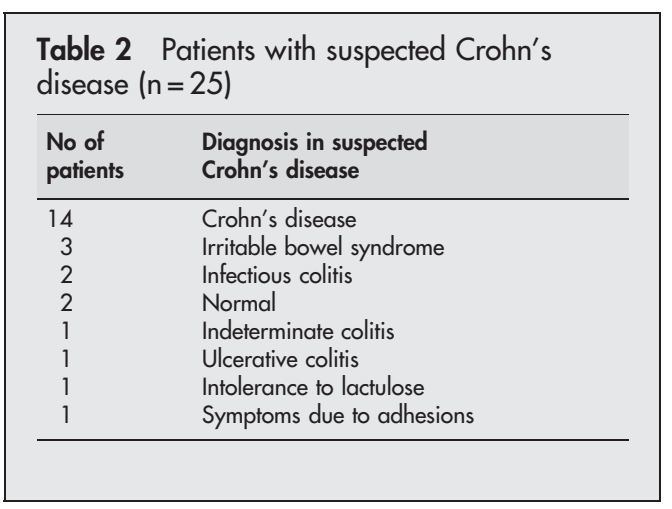

Table 3 Patients with suspected small bowel Crohn's disease: sensitivity and specificity for "Crohn-like" small bowel lesions for direct comparison of magnetic resonance imaging (MRI) with capsule endoscopy (CE)

\begin{tabular}{lll}
\hline & Sensitivity & Specificity \\
\hline CE & $12 / 13(92 \%)$ & $10 / 10(100 \%)$ \\
MRI & $10 / 13(77 \%)$ & $8 / 10(80 \%)$ \\
\hline$p>0.05 ;$ kappa $=0.57$. &
\end{tabular}

No small bowel lesions were found in the remaining 12 patients. MRI detected small bowel pathology indicative of Crohn's disease in 10/13 (77\%) patients. Non-specific contrast enhancement was reported in four patients with a final diagnosis of Crohn's disease (false negative findings). Bowel wall thickening and some enhancement of the small bowel was regarded as indicative of Crohn's disease in two cases but endoscopy (including CE) detected no mucosal lesions (false positive findings). No pathology was detected in the remaining patients who were diagnosed as not suffering from Crohn's disease.

In $\mathrm{CE}$, small bowel lesions were found in 12 of 13 patients $(92 \%)$ and in none of the patients where a diagnosis of Crohn's disease was finally rejected. Patchy erythema in the proximal and distal small bowel segments described by CE in another patient were regarded as non-specific. In this patient, repeated colonoscopy disclosed ulcerous lesions suggesting active Crohn's disease of the terminal ileum and colon. Therefore, results of $\mathrm{CE}$, much like those of MRI, were regarded as false negative (table 3 ).

CE was the exclusive diagnostic tool in two patients. MRI showed discontinuous small bowel enhancement after application of contrast medium but no bowel wall thickening (non-specific finding) in one case while CE demonstrated multiple $(>40)$ aphthous ulcers of the upper and middle small bowel. In the other patient, MRI was also non-specific but CE confirmed upper small bowel ulcerations, indicating Crohn's disease. In both patients, anti-inflammatory therapy was initiated and symptoms improved.

\section{Comparison of imaging methods}

Enteroclysis was less sensitive than MRI (enteroclysis $v$ MRI, $\mathrm{p}=0.011$ ) and CE (enteroclysis $v \mathrm{CE}, \mathrm{p}=0.002$ ) in direct comparison of all patients investigated. CE was slightly more sensitive than MRI but this marginal difference did not reach statistical significance. Details on the diagnostic yield of enteroclysis, MRI, and CE in small bowel Crohn's disease are given in table 4. 
Table 4 Performance of investigational methods in detecting small bowel Crohn's disease in patients with previously established diagnosis Crohn's disease and with suspected Crohn's disease

\begin{tabular}{lcrr}
\hline & Enteroclysis & MRI & CE \\
\hline Established CD & 16 & 22 & 13 \\
Findings & 8 & 3 & 1 \\
Normal & 67 & 88 & 93 \\
Detection rate (\%) & 3 & 2 & 13 \\
Drop-out & 27 & 27 & 27 \\
$\quad$ Total & & & \\
Suspected CD & 4 & 10 & 12 \\
$\quad$ True positive & 0 & 2 & 0 \\
False positive & 6 & 8 & 11 \\
True negative & 6 & 4 & 1 \\
False negative & 100 & 83 & 100 \\
PPV (\%) & 50 & 66 & 92 \\
NPV (\%) & 9 & 1 & 1 \\
Drop-out & 25 & 25 & 25 \\
Total & & & \\
\hline
\end{tabular}

$\mathrm{CD}$, Crohn's disease; $\mathrm{MRI}$, magnetic resonance imaging; $\mathrm{CE}$, capsule endoscopy; PPV, positive predictive value; NPV, negative predictive value.

\section{Follow up data in patients with suspected Crohn's disease}

Follow up data were available in 22 of 25 patients (88\%) with suspected Crohn's disease while three were lost. Mean follow-up time was 14.5 (SD 6.5) months. The diagnosis of Crohn's disease remained unchanged in all cases. The diagnosis of ulcerative colitis (one patient) was revised to "indeterminate colitis". Other diagnoses were confirmed by follow up.

\section{Procedure related events}

Procedure related events occurred in six patients (CE $n=1$, MRI $n=3$, and enteroclysis $n=2$ ). Ingestion of the capsule was followed by colicky abdominal pain four hours later in one case. A plain abdominal radiograph showed the capsule in the right lower abdominal quadrant. CE imaging data disclosed inflammatory lesions in the upper small bowel and high grade luminal narrowing in an inflamed region of the distal small bowel. The capsule was retained proximal to this bowel stricture but did not cause complete obstruction or ileus. After intravenous corticosteroids were started, symptoms improved but did not resolve until approximately 72 hours later when the capsule was excreted. Surgical intervention was not necessary, neither during this hospital

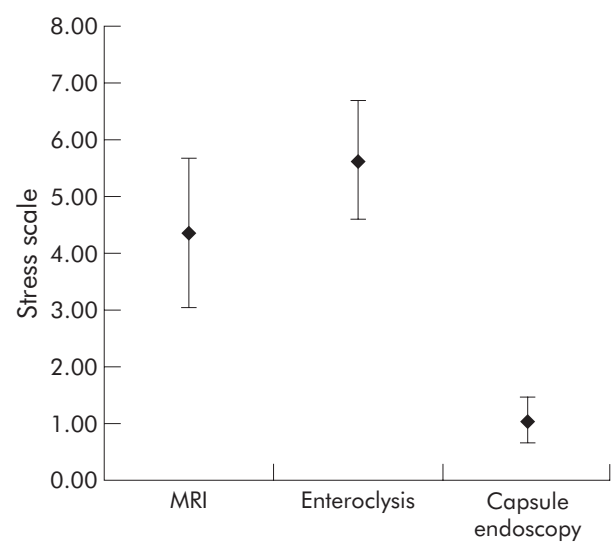

Figure 4 Patients' acceptance of the investigation. Psychological stress during the investigation on a scale from 0 (no stress) to 10 (unbearable stress). $n=22$. stay nor later in the course of follow up. Abdominal ultrasound and enteroclysis had failed to detect this stricture.

In two patients, acquisition of MRI data was incomplete due to claustrophobia; one patient refused an MRI examination. Two patients did not tolerate placement of the transnasal tube for enteroclysis.

\section{Predicting stricturing disease}

In 14 patients, significant bowel strictures were detected by MRI and/or enteroclysis. Concordance of MRI and enteroclysis in discovering strictures was high in retrospective analysis (kappa $=0.83)$. Eight of these patients had been operated on 3-14 years (mean 6.2 (SD 4.1) years) earlier. In five of the eight, the stricture affected the anastomosis while three had recurring stricturing disease at other locations. Previous surgery $(8 / 14 v 6 / 38 ; p=0.005)$, leading symptom "abdominal pain" (13/14 v 14/38; $<<0.001)$, and a previously established diagnosis of Crohn's disease (13/14 v 14/38; $\mathrm{p}<0.001$ ) were identified as prognostic factors.

\section{Patient comfort}

Twenty two patients returned the questionnaire; all had been investigated by all three methods. Mean MRI stress grade was 4.4 (SD 2.95) and was regarded as less stressful than enteroclysis (stress grade $5.6(2.38) ; \mathrm{p}=0.02$ ). CE (stress grade $1.1(0.9))$ was found to be significantly less stress associated than both MRI and enteroclysis $(p<0.001$, respectively; fig 4).

\section{DISCUSSION}

To the best of our knowledge, the present study is the first to prospectively compare CE with MRI and double contrast fluoroscopy (small bowel enteroclysis) in patients with suspected small bowel Crohn's disease. While enteroclysis was found to be the least sensitive modality, CE was only slightly more sensitive than MRI ( $12 v 10$ of 13 in suspected Crohn's disease and $13 v 11$ of 14 in established Crohn's disease), and this small difference did not achieve statistical significance. One would have to perform a trial on 93 (power $80 \%$, alpha 0.05 ) or 120 patients (power 90\%, alpha 0.05 ) with Crohn's disease of the small bowel in order to statistically prove or disprove such a small advantage in sensitivity of $14 \%(92 \% \vee 78 \%)$. Even if such marginal superiority of CE could be established, it would most probably not alter diagnostic decision making in the individual patient, as discussed below.

\section{Methodological considerations}

One of the problems inherent in diagnostic studies in small bowel Crohn's disease is the lack of a non-surgical "gold standard" for comparison. Assuming that the apparently most sensitive method should set the "standard" may be incorrect due to unrecognised false positive results. For this reason, we used a combined diagnostic end point composed of all imaging methods, including ileocolonoscopy as well as clinical and laboratory data and evolution of the diagnosis during follow up. Thus our rating of CE, MRI, and conventional fluoroscopic enteroclysis was as objective as possible.

Another potential source of error in such studies is the observation of non-specific lesions even in healthy subjects, which could be falsely diagnosed as indicative of Crohn's disease. We tried to avoid this problem by defining as narrowly as possible the distinction between "non-specific" and "diagnostic" for Crohn's disease on the basis of our previous experience with CE und MRI, and of findings described in the literature. Furthermore, investigators were blinded to the results of the various competing imaging methods. 
Finally, our results were obtained in a highly preselected group of patients. However, there was no apparent reason to assume that the hierarchy of diagnostic efficacy of the three imaging methods investigated should be substantially altered in a population showing a smaller prevalence of Crohn's disease. If anything, the specificity of positive results of all three methods, possibly most prominently for CE, would decrease if the number of subjects with non-specific findings were to increase.

The results concerning enteroclysis in suspected Crohn's disease might have been hampered by the fact that some patients refused this procedure, mainly due to fear of radiation. While this is correct theoretically, enteroclysis was still less sensitive than MRI (enteroclysis $v$ MRI, $\mathrm{p}=0.011$ ) and CE (enteroclysis $v \mathrm{CE}, \mathrm{p}=0.002$ ) in the subgroup of patients in whom all three methods had been applied. Similar results have been reported. ${ }^{13}$ In a trial of 84 patients, sensitivity was $85.4 \%$ and $95.2 \%$, and specificity was $76.9 \%$ and $92.6 \%$ for enteroclysis and MRI, respectively. Others found similar sensitivities for conventional enteroclysis and MR-enteroclysis in detecting small bowel Crohn's disease. ${ }^{14}$

In addition, we believe that refusal to undergo imaging because of problems inherent in the examination itself should not be disregarded when calculating diagnostic efficacy.

\section{Diagnostic value of $C E, M R I$, and enteroclysis}

In the pre-CE era, results of intraoperative endoscopy of the entire small bowel increased the number of findings over those known preoperatively in $20-35 \%{ }^{15}{ }^{16}$ and influenced surgical interventions in approximately $60 \%{ }^{17}$ of patients with Crohn's disease requiring surgical intervention. Information on the comparative diagnostic efficacy of CE in Crohn's disease is sparse to date. In early more or less anecdotal reports, detection rates of CE were reported as 70\% (12 of 17 Crohn lesions) ${ }^{7}$ and $42.8 \%$ (9 of 21$)^{18}$ in patients with an unspecified but apparently non-diagnostic prior investigational workup. The diagnostic yield of CE in explaining the symptoms of abdominal pain, diarrhoea, and weight loss was found to be higher $(70 \%)$ than that of small bowel follow through and enteric computed tomography (35\%) in another study. ${ }^{19}$ However, final diagnoses were not given in this report leaving the diagnostic efficacy of CE for Crohn's disease unknown. Other feasibility studies of CE were mostly restricted to patients with suspected Crohn's disease, ${ }^{20}$ and CE was not formally compared with other investigations. ${ }^{21} 22$

Enteroclysis or small bowel follow through have been standard radiological investigations to detect small bowel Crohn's disease until now, but the preference for one or the other still depends on institutional standards. ${ }^{23}{ }^{24}$ In a recent study, CE was compared with barium contrast small bowel follow-through (SBFT) in 30 patients with a previously established diagnosis of Crohn's disease..$^{25}$ Although active small bowel Crohn's disease was detected in a similar proportion of patients by both methods (CE 21/30 patients; SBFT 20/30), findings were inconsistent in about $35 \%$ of patients: in six patients, CE found lesions that had escaped detection by SBFT while SBFT was diagnostic in five patients in whom CE had failed. In a retrospective study ${ }^{26}$ of 40 patients with $\mathrm{CE}$, three were found to have significant small bowel ulceration despite normal fluoroscopic enteroclysis. In the present study, the sensitivity of enteroclysis was found to be significantly inferior to both CE and MRI. As radiation exposure is significant ${ }^{27}$ and diagnostic capability for extraluminal disease limited, fluoroscopic enteroclysis should be abandoned as a diagnostic tool for inflammatory bowel disease if the modern MRI technique is available.
After completion of this study, a prospective comparison of $\mathrm{CE}$ with computed tomography enteroclysis ${ }^{28}$ in patients with an established diagnosis of Crohn's disease was published. Small bowel lesions were detected by CE in 25 of 41 patients compared with only 12 of 41 with computed tomography (CT) enteroclysis $(p=0.004)$. Mucosal lesions escaped CT in 13 cases. In the present study, MRI (78\%) was far more sensitive in detecting Crohn's lesions of the small bowel than CT enteroclysis in the above mentioned investigation (12 of 25: $48 \%$ ). Although the patient groups in the two studies may not be compared directly, lack of radiation exposure and greater diagnostic sensitivity strongly support the use of MRI rather than CT technology for investigating the small bowel in Crohn's disease. Furthermore, MRI is widely applied in perianal fistulising Crohn's disease, ${ }^{29}{ }^{30}$ and detection of intestinal and extraintestinal (abscesses, fistulae, mesenteric lymph nodes) manifestations of Crohn's disease is feasible. ${ }^{14}$

Not surprisingly, patients preferred CE to fluoroscopic enteroclysis. However, the use of CE as a first line diagnostic measure for small bowel Crohn's disease is limited by potential capsule retention with the risk of "non-natural excretion". While in some reports no side effects of CE were observed in an accumulated 58 cases, ${ }^{78}{ }^{18}$ recent publications suggest capsule retention occurs in 5\% to more than $10 \%$ of patients, ${ }^{20-22}{ }^{25}$ even in those with only suspected disease. An even higher index of suspicion is warranted in patients with established Crohn's disease: in the present study, strictures were detected in $48 \%$ of these patients. Therefore, CE should only be applied when strictures have been excluded by the most reliable method available which, according to the present results, is MRI.

\section{Which examination to see what in clinical practice?}

In conclusion, $\mathrm{CE}$ is highly sensitive in diagnosing small bowel Crohn's disease and well tolerated by the patients. It may even be slightly superior to MRI, but this potential advantage is relevant in a few cases in clinical practice only. Imaging of the small bowel in patients with suspected or established Crohn's disease is indicated for the initial determination of the extent of bowel involvement, if a diagnosis cannot be made by upper endoscopy and ileocolonoscopy, and if demonstration or exclusion of small bowel involvement would affect medical or surgical therapy. In this situation, the first most simple and quite helpful examination is abdominal ultrasound. As the next step, if questions of therapeutic relevance remain, MRI should be performed to provide clues to both intestinal and extraintestinal Crohn's disease, although it may lack the superior sensitivity of CE in detecting minor lesions limited to the mucosa. Using this algorithm, CE will most probably be applicable to no more than $10 \%$ of the population in which the small bowel should be specifically visualised. In the present trial of 52 patients, this applied to four, in whom CE was the only effective diagnostic measure.

\section{ACKNOWLEDGEMENTS}

The authors are greatly indebted to Ms S Behl for her biostatistical contribution, and to Ms Chr Zschorn and Mr M Neugebauer for technical support.

\section{Authors' affiliations}

J G Albert, F Martiny, A Krummenerl, J Leßke, C M Göbel, E Lotterer, H H Nietsch, W E Fleig, First Department of Medicine, Martin-LutherUniversity, Halle (Saale), Germany

K Stock, C Behrmann, Department of Radiology, Martin-LutherUniversity, Halle (Saale), Germany

Conflict of interest: None declared. 
The study was presented in part at the 12th United European Gastroenterology Week, Prague, Czech Republic, 25-29 September 2004, and was published as an abstract (Gut 2004;53:S4, A2).

\section{REFERENCES}

1 Hanaver SB. The clinical presentation and diagnosis of inflammatory bowel disease. In: Satsangi J, Sutherland LR, Colombel JF, et al. Inflammatory bowel disease. Philadelphia: Elsevier Ltd, 2003:167-8.

2 Iddan G, Meron G, Glukhovsky A, et al. Wireless capsule endoscopy. Nature 2000:405:417.

3 Rhodes Al, Shorvon PJ. Recent advances in small-bowel imaging: a review. Curr Opin Gastroenterol 2001;17:132-9.

4 Maccioni F, Visciodi A, Broglia L, et al. Evaluation of Crohn disease activity with magnetic resonance imaging. Abdom Imaging 2000;25:219-28.

5 Ell C, Remke S, May A, et al. The first prospective controlled trial comparing wireless capsule endoscopy with push enteroscopy in chronic gastrointestina bleeding. Endoscopy 2002;34:685-9.

6 Mylonaki M, Fritscher-Ravens A, Swain P. Wireless capsule endoscopy: a comparison with enteroscopy. Gut 2003;52:1122-6.

7 Fireman Z, Mahaina E, Broide E, et al. Diagnosing small bowel Crohn's disease with wireless capsule endoscopy. Gut 2003;52:390-2

8 Schulmann K, Hollerbach S, Schmiegel W. Diagnosing small bowel Crohn's disease with wireless capsule endoscopy. Gut 2003;52:1531-2.

9 Eliakim R, Suissa A, Yassin K, et al. Wireless capsule video endoscopy compared to barium follow-through and computerized tomography in patients with suspected Crohn's disease - final report. Dig Liver Dis 2004;36:519-22.

10 Ginsberg GG, Barkun AN, Bosco JJ, et al. ASGE technology status evaluation report: Wireless capsule endoscopy. Gastrointest Endosc 2002;56:621-4.

11 Albert J, Göbel CM, Leßke J, et al. Simethicone for small bowel preparation for capsule endoscopy: a systematic, single-blinded, controlled study. Gastrointest Endosc 2004:59:487-91.

12 Herlinger HA. A modified technique for the double contrast small bowel enema. Gastrointest Radiol 1978;3:201-7.

13 Rieber A, Wruk D, Potthast S, et al. Diagnostic imaging in Crohn's disease: comparison of magnetic resonance imaging and conventional imaging methods. Int J Colorectal Dis 2000;15:176-81.

14 Schreyer AG, Seitz J, Feuerbach S, et al. Modern imaging using computer tomography and magnetic resonance imaging for inflammatory bowel disease. Inflamm Bowel Dis 2004;10:45-54.
15 Lescut $D$, Vanco $D$, Bonniere $P$, et al. Perioperative endoscopy of the whole small bowel in Crohn's disease. Gut 1993;34:647-9.

16 Esaki M, Matsumoto T, Hizawa K, et al. Intraoperative enteroscopy detects more lesions but is not predictive of postoperative recurrence in Crohn's disease. Surg Endosc 2001:15:455-9.

17 Smedh K, Olaison G, Nystrom PO, et al. Intraoperative enteroscopy in Crohn's disease. Br J Surg 1993;80:897-900.

18 Herrerias JM, Caunedo A, Redriguez-Téllez M, et al. Capsule endoscopy in patients with suspected Crohn's disease and negative endoscopy. Endoscopy 2003;35:564-8.

19 Eliakim R, Fischer D, Suissa A, et al. Wireless capsule video endoscopy is a superior diagnostic tool in comparison to barium follow-through and computerized tomography in patients with suspected Crohn's disease. Eur J Gastroenterol Hepatol 2003;15:363-7.

$20 \mathrm{Ge} \mathrm{ZZ}$, Hu YB, Xiao SD. Capsule endoscopy in diagnosis of small bowel Crohn's disease. World J Gastroenterol 2004;10:1349-52.

21 Reddy DN, Kaffes AJ, Sriram PVJ, et al. Capsule endoscopic features of Crohn's disease. Dig Endosc 2004;16:138-42.

22 Mow WS, Lo SK, Targan SR, et al. Initial experience with wireless capsule enteroscopy in the diagnosis and management of inflammatory bowel disease. Clin Gastroenterol Hepatol 2004;2:31-40.

23 Kelvin FM, Maglinte DD. Enteroclysis or small bowel follow through in Crohn's disease? Gastroenterology, 1998;114, 1349-51.

24 Bernstein CN, Boult IF, Greenberg HM, et al. A prospective randomized comparison between small bowel enteroclysis and small bowel follow-through in Crohn's disease. Gastroenterol 1997; 113:390-8.

25 Buchman AL, Miller FH, Wallin A, et al. Videocapsule endoscopy versus barium contrast studies for the diagnosis of Crohn's disease recurrence involving the small intestine. Am J Gastroenterol 2004:99:2171-7.

26 Liangpunsakul S, Chadalawada V, Rex DK, et al. Wireless capsule endoscopy detects small bowel ulcers in patients with normal results from state of the art enteroclysis. Am J Gastroenterol 2003;98:1295-8.

27 Mahesh M. Fluoroscopy: patient radiation exposure issues. Radiographics $2001 ; 21: 1033-45$

28 Voderholzer WA, Beinhoelzl J, Rogalla P, et al. Small bowel involvement in Crohn's disease: a prospective comparison of wireless capsule endoscopy and computed tomography enteroclysis. Gut 2005;54:369-73.

29 Buchanan G, Halligan S, Williams A, et al. Effect of MRI on clinical outcome of recurrent fistula-in-ano. Lancet 2002;360:1661-2.

30 Morris J, Spencer JA, Ambrose NS. MR imaging classification of perianal fistulas and its implications for patient management. Radiographics 2000;20:623-35 